\title{
Outcomes of hospital-acquired SARS-CoV-2 infection in the Canadian first wave epicentre: a retrospective cohort study
}

\author{
Eve Melançon MD, Marc Brosseau MD, Anthony Bartoli MD PharmD, Annie-Claude Labbé MD, \\ Christian Lavallée MD MSc, Xavier Marchand-Senécal MD, Han Ting Wang MD MSc
}

Abstract

Background: During the first wave of the COVID-19 pandemic, a substantial number of Quebec hospitals were hit by hospital-acquired (HA) SARS-CoV-2 infections. Our objective was to assess whether mortality is higher in HA cases than in non-hospital-acquired (NHA) cases and determine the prevalence of HA-SARS-CoV-2 infection in our hospital.

Methods: This retrospective single-centre cohort study included all adults ( $\geq 18 \mathrm{yr}$ ) who had COVID-19, admitted to Hôpital Maisonneuve-Rosemont (Montréal, Canada) from Mar. 1 to June 30, 2020. We collected data on demographic characteristics, comorbidities, treatment, admission to the intensive care unit (ICU) and mechanical ventilation requirements from electronic health records. We adjudicated hospital acquisition based on the timing of symptom onset, and polymerase chain reaction testing for and exposures to SARS-CoV-2. To evaluate the association between HA-SARS-CoV-2 infection and in-hospital mortality, we computed a multivariable logistic regression analysis including known risk factors for death in patients with COVID-19 as covariates.

Results: Among 697 patients with SARS-CoV-2 infection, $253(36.3 \%)$ were classified as HA. The mortality rate was higher in the HA group than in the NHA group (39.1\% v. 25.9\%, $p=0.001)$, while the rates of ICU admission $(8.3 \% \mathrm{v} .19 .1 \%, p=0.001)$ and requirement for mechanical ventilation $(3.6 \% \mathrm{v} .13 .0 \%, p=0.001)$ were lower. Multivariable logistic regression analysis showed that HA-SARS-CoV-2 infection in patients younger than 75 years is an independent risk factor for death (odds ratio $2.78,95 \%$ confidence interval 1.44-5.38).

Interpretation: Our results show that HA-SARS-CoV-2 infection in younger patients was associated with higher mortality. Future studies need to evaluate relevant patient-centred long-term outcomes in this population.

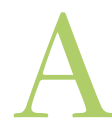

s of January 2022 in Canada, about 2300000 cases of COVID-19 have been confirmed since the beginning of the pandemic, with more than 30500 deaths. ${ }^{1}$ During the first wave of this pandemic (first half of 2020), the province of Quebec, whose population was nearly 8.5 million as of January 2021, ${ }^{2}$ had the highest number of COVID-19 cases compared with other provinces. Montréal was the epicentre, accounting for more than one-third of all cases in the province. ${ }^{3}$ In the first wave of the pandemic, about $10 \%$ of patients who developed COVID-19 in Wuhan, China, required admission to hospital and $5 \%$ required admission to the intensive care unit (ICU)., ${ }^{4,5}$ Older patients and those with existing comorbidities are at higher risk of adverse outcomes. ${ }^{5}$

Amid this pandemic, hospitals have tried to continue their usual activities and provide urgent care. Unfortunately, hospital admission represents a potential environment for viral transmission to vulnerable patients. ${ }^{6}$ As of February 2021, there were mixed data about outcomes for patients with hospital-acquired (HA) SARS-CoV-2 infection compared with non-hospital-acquired (NHA) infection, as well as inhospital transmission dynamics of SARS-CoV-2. Some studies showed a case fatality rate as high as $36 \%$ for patients with HA-COVID-19, ${ }^{7}$ while others reported a mortality rate lower than that of patients with NHA-COVID- $19 .{ }^{8}$

Therefore, we aimed to assess whether mortality and complications were increased in HA cases of SARS-CoV-2 infection when compared with NHA cases at Hôpital MaisonneuveRosemont. We also explored the role of patients sharing multi-bed rooms in hospital with respect to in-hospital transmission of SARS-CoV-2.

\section{Competing interests: None declared.}

This article has been peer reviewed.

Correspondence to: Han Ting Wang, ht.wang85@gmail.com CMAJ Open 2022 February 1. DOI:10.9778/cmajo.20210055 


\section{Methods}

\section{Study design and setting}

We conducted a retrospective cohort study through chart review at Hôpital Maisonneuve-Rosemont - a tertiary academic hospital built in 1954 and hosting 544 beds, including 16 ICU beds - in Montréal, Canada. On Mar. 20, 2020, it was designated as a COVID-19 care centre by the Quebec Ministry of Health and Social Services, and thereafter received transfers from other institutions. Before this date, most patients with COVID-19 who presented to our centre had been transferred to other hospitals. During the first wave of the pandemic (Jan. 25 to June 30, 2020), up to 191 designated beds for patients with confirmed COVID-19 were made available in our centre, including 19 ICU beds. We followed the Strengthening the Reporting of Observational Studies in Epidemiology (STROBE) reporting guidelines. ${ }^{9}$

\section{Participants}

Our analysis included all patients with confirmed SARSCoV-2 infection aged 18 years or older who had been admitted to our hospital from Mar. 1 to June 30, 2020. We excluded patients with a diagnosis of COVID-19 more than 2 weeks before hospital admission. The list of all patients with SARS-CoV-2 infection was provided by the hospital Infection Prevention and Control (IPAC) division, which prospectively tracked all inpatients and outpatients positive for the virus via polymerase chain reaction (PCR) testing in our institution, based on the local laboratory data and data from other institutions for patient transfers. The IPAC division includes certified infection control practitioners, supervised by a medical director. We then cross-matched this list with our hospital administrative data set to determine which patients had been admitted to hospital.

\section{Data sources}

We created and managed a data abstraction form using Research Electronic Data Capture (REDCap). ${ }^{10}$ Two senior residents (A.B. and E.M.) and 2 research nurses performed data collection. The 2 residents supervised the research nurses throughout the project and performed accuracy checks throughout data collection. Although we did not perform formal reliability testing, most errors occurred only during the first weeks of the collection process.

We gathered data on demographic characteristics, place of origin before hospital admission, deprivation index based on postal code, ${ }^{11}$ and comorbidities based on the Charlson Comorbidity Index score, ${ }^{12}$ as well as the need for mechanical ventilation and ICU admission. We also collected information on specific drug therapies, such as hydroxychloroquine, azithromycin, tocilizumab, corticosteroids (defined as any dose higher than adrenal insufficiency supplementation dose) and lopinavir-ritonavir. We did not compile information on anticoagulation prescriptions, as this drug class was not considered as a potential therapy at the time of data collection.

\section{Case definitions}

SARS-CoV-2 infection was defined as at least 1 positive SARS-CoV-2 PCR test (combined throat and nasopharyngeal swab or lower respiratory tract aspiration). We used the date of the first positive test if multiple tests were performed. We then classified cases as HA or NHA using definitions from the Institut National de Santé Publique du Québec (INSPQ). ${ }^{13}$ Proven HA cases were defined as a positive SARS-CoV-2 PCR test more than 14 days after hospital admission or when an in-hospital epidemiologic link was identified with a person known by the IPAC division to have SARS-CoV-2 infection. The medical director of IPAC made all final attributions based on epidemiologic links, using best clinical judgment without being aware of each patient's outcome. We defined suspected HA cases as occurring 7 to 14 days after hospital admission. We defined NHA cases as those occurring fewer than 7 days after hospital admission, including infections acquired in the community and longterm care facilities or any other congregate living settings. For our analysis, we combined proven and suspected HA cases as HA cases, to match other published studies. ${ }^{7,14}$

\section{Infection prevention and control and testing practices}

Infection prevention and control practices, visitor restrictions and testing practices changed during the pandemic's first wave, reflecting evolving knowledge and scaling-up of SARS-CoV-2 PCR testing availability (Appendix 1, eTable 1, available at www.cmajopen.ca/content/10/1/E74/ suppl/DC1). Patients with COVID-19 and those admitted for another reason who were also found to be positive for SARS-CoV-2 infection were admitted to designated COVID-19 wards.

Droplet and contact precautions were required when caring for patients with suspected or confirmed SARS-CoV-2 infection, with personal protective equipment (PPE) including procedure mask, eye protection, isolation gown and gloves. N95 respirators were reserved for aerosol-generating medical procedures or patients presenting with predefined severity criteria concordant with INSPQ guidelines. However, our hospital was affected by PPE and hand sanitizer shortages, leading to a suboptimal uptake of some IPAC practices. Symptomatic health care workers and those with high levels of exposure to a person known to be positive for SARS-CoV-2 infection were asked to stay home, received testing and were managed by the Occupational Health and Safety Division.

All patients admitted without SARS-CoV-2 infection with high levels of exposure to a person positive for SARS-CoV-2 infection were prospectively flagged by IPAC as close contacts and followed a 14-day quarantine under droplet and contact precautions, with daily symptom monitoring and, from late April 2020, repeated PCR testing. When COVID-19 outbreaks occurred on wards, additional measures were initiated: high-touch surface disinfection, restricted patient transfers, implementation of droplet and contact precautions for all patients, and SARS-CoV-2 PCR testing if symptoms developed, and from Apr. 21, repeated testing of all patients even if asymptomatic. 


\section{Outcomes}

The primary clinical outcome was in-hospital mortality. Secondary clinical outcomes were dispositions at discharge, and hospital readmission rates up to 90 days after discharge. Additionally, we looked at the prevalence of patients with HASARS-CoV-2 infection and acquisition of infection in patients who shared a multi-bed room with patients with known SARS-CoV-2 infection during their period of contagiousness (from $2 \mathrm{~d}$ before symptom onset to $10 \mathrm{~d}$ after). Acquisition of SARS-CoV-2 infection was linked to viral transmission between patients in the same room only if close contacts developed symptoms (or tested positive if asymptomatic) 3 or more days after symptom onset in the index patient and their first contact, and up to 14 days after their last contact. We used a 3-day gap as it represents the lower end of the SARS$\mathrm{CoV}-2$ serial interval. ${ }^{15}$

\section{Statistical analysis}

We summarized continuous variables as median with the interquartile range (IQR), and categorical variables as proportions. We compared outcomes between the HA and NHA groups using $\chi^{2}$ test for categorical data. To evaluate the association between HA-SARS-CoV-2 infection and in-hospital mortality, we performed a multivariable logistic regression by including the following prespecified risk factors for death in patients with SARS-CoV-2: age, sex, moderate to severe chronic renal disease, solid tumour, hematologic malignancy, diabetes, chronic obstructive pulmonary disease (COPD) and myocardial infarction. Considering the potential interaction between age and HA status on mortality, we calculated agegroup-specific odds ratios for HA-SARS-CoV-2 infection.
This was further supported by the presence of interaction on univariate analysis. We quantified the degree of collinearity using variance inflation factors. As a sensitivity analysis, we performed the same analysis for only proven HA cases after excluding all suspected HA cases. We used STATA MP version 16.1 for all analysis.

\section{Ethics approval}

The Research Ethics Board of the Centre intégré universitaire de santé et de services sociaux (CIUSSS) de l'Est-de-l'Île-deMontréal approved our study protocol.

\section{Results}

During the 3-month period, 734 patients were screened for inclusion and 37 were excluded, resulting in 697 patients either admitted to hospital with COVID-19 or who had acquired SARS-CoV-2 infection while in hospital (Figure 1). The first admission of a patient with COVID-19 was on Mar. 19, 2020, and the first patient with HA-SARS$\mathrm{CoV}-2$ infection became symptomatic on Mar. 24 (epidemic curve shown in Figure 2). A total of 253 (36.3\%) patients with HA-SARS-CoV-2 infection were classified as being HA (proven: 217 [85.4\%]; suspected: 36 [14.6\%]). Among patients with proven HA-SARS-CoV-2, 101 $(46.5 \%)$ had a positive SARS-CoV-2 PCR test more than 14 days after admission; the 116 others had an epidemiologic link identified (see Appendix 1, eFigure 1, for timing of diagnosis from admission day). Out of 139 identified close-contact patients associated with sharing a multi-bed room with a patient with COVID-19, 45 (32.4\%) developed

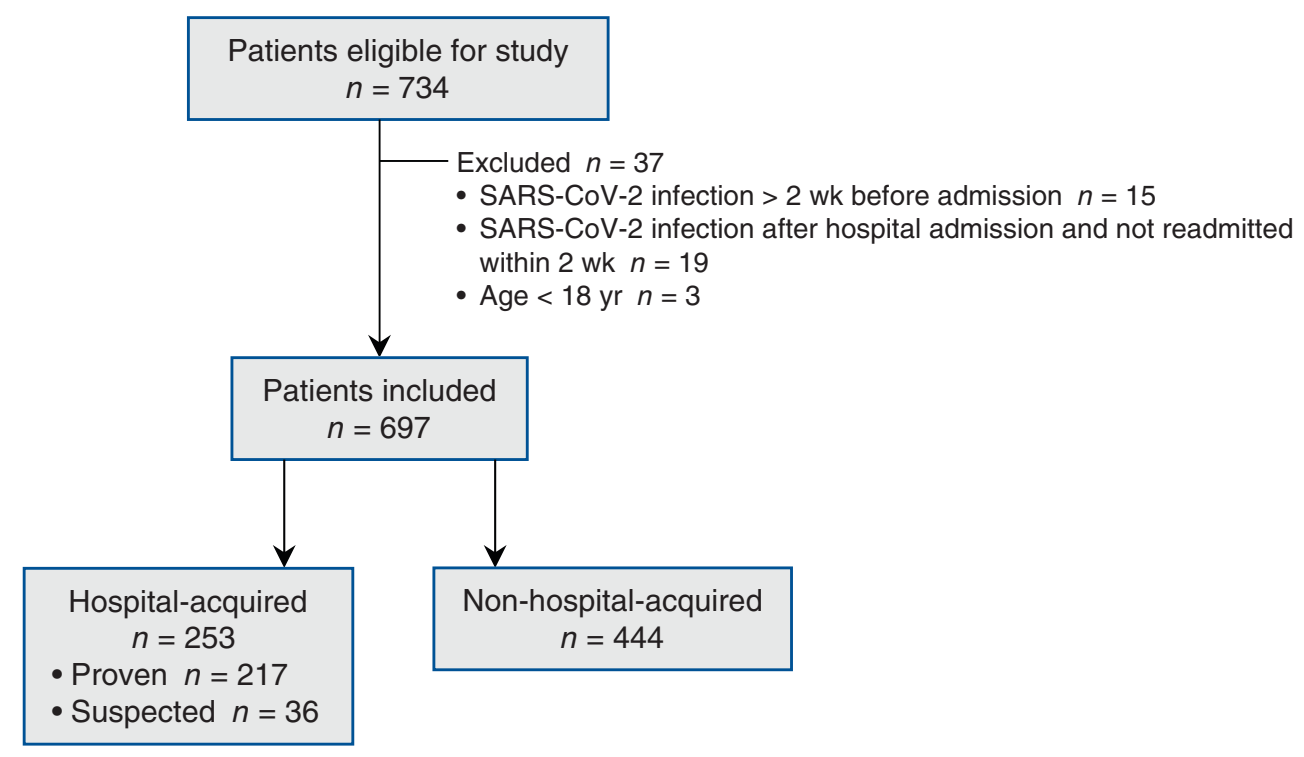

Figure 1: Flow chart of included patients with SARS-CoV-2 infection in hospital. 
SARS-CoV-2 infection while in hospital, and 6 had a positive SARS-CoV-2 PCR test with an unknown date of symptom onset within 14 days after discharge (3 within 7 d). We classified 444 patients as NHA, comprising 406 $(91.2 \%)$ community-acquired cases.

The median age was 75 years (IQR 62-85) for the full cohort and $356(51.1 \%)$ patients were female (Table 1). Age younger than 75 years was less frequent in the HA group $(n=98$ [38.7\%], $p=0.003)$ than in the NHA group $(n=231$ [52.0\%]). Patients with HA-SARS-CoV-2 infection had a higher previous 5 -year history of cancer (localized $n=49$ [19.4\%], HA group v. $n=39$ [8.8\%], NHA group; metastatic $n=28$ [11.1\%], HA group v. $n=14$ [3.2\%], NHA group; $p<0.001)$ and COPD $(n=60$ [23.7\%], HA group v. $n=65[14.6 \%]$, NHA group; $p=0.003)$. Surgical procedures were performed more frequently in patients with HA-SARS-CoV-2 infection than in patients with NHA infection $(n=37$ [14.6\%] v. $n=13[2.9 \%] ; p=0.001)$. Corticosteroid treatment was prescribed in $46(18.2 \%)$ patients with HA infection and $66(14.9 \%)$ patients with NHA infection $(p=0.25)$. Two patients with HA-SARS$\mathrm{CoV}-2$ infection received tocilizumab and 3 patients with NHA infection received hydroxychloroquine. Both treatments were not recommended for COVID-19 at the time of our study.

\section{Clinical outcomes}

Patients with HA-SARS-CoV-2 infection had a higher rate of in-hospital mortality $((n=99[39.1 \%])$ than patients with NHA infection ( $n=115$ [25.9\%], $p=0.001)$, with lower rates of ICU admission and requirement for mechanical ventilation $(n=21[8.3 \%]$ v. $n=85[19.1 \%], p<0.001$ and $n=9$ $[3.6 \%]$ v. $n=58[13.0 \%], p=0.001$, respectively) (Table 2). Most patients with SARS-CoV-2 infection in the ICU admitted within 7 days of a positive test $(n=17$ [81.0\%] in the HA group v. $n=74[87.1 \%]$ in the NHA group, $p=$ 0.47). Patients who were discharged after HA-SARS-CoV-2 infection were less likely to be discharged directly home and were more likely to be transferred to a rehabilitation centre than patients with NHA infection $(p=0.001)$. Readmissions within 90 days after discharge occurred more frequently in the HA group than in the NHA group $(n=24$ [15.3\%] v. $n=13[4.0 \%], p=0.001)$.

After multivariable analysis, patients younger than 75 years with HA-SARS-CoV-2 infection had increased odds of death over patients with NHA infection in hospital (odds ratio [OR] $2.78,95 \%$ confidence interval $[\mathrm{CI}]$ 1.44-5.38) (Table 3). There was no increased risk of death for older patients (OR $1.25,95 \%$ CI $0.64-2.42$ in the 75-84-yr age group and OR $0.75,95 \%$ CI $0.41-1.35$ in the $\geq 85$-yr age group) compared with patients younger than 75 years. Moderate to severe

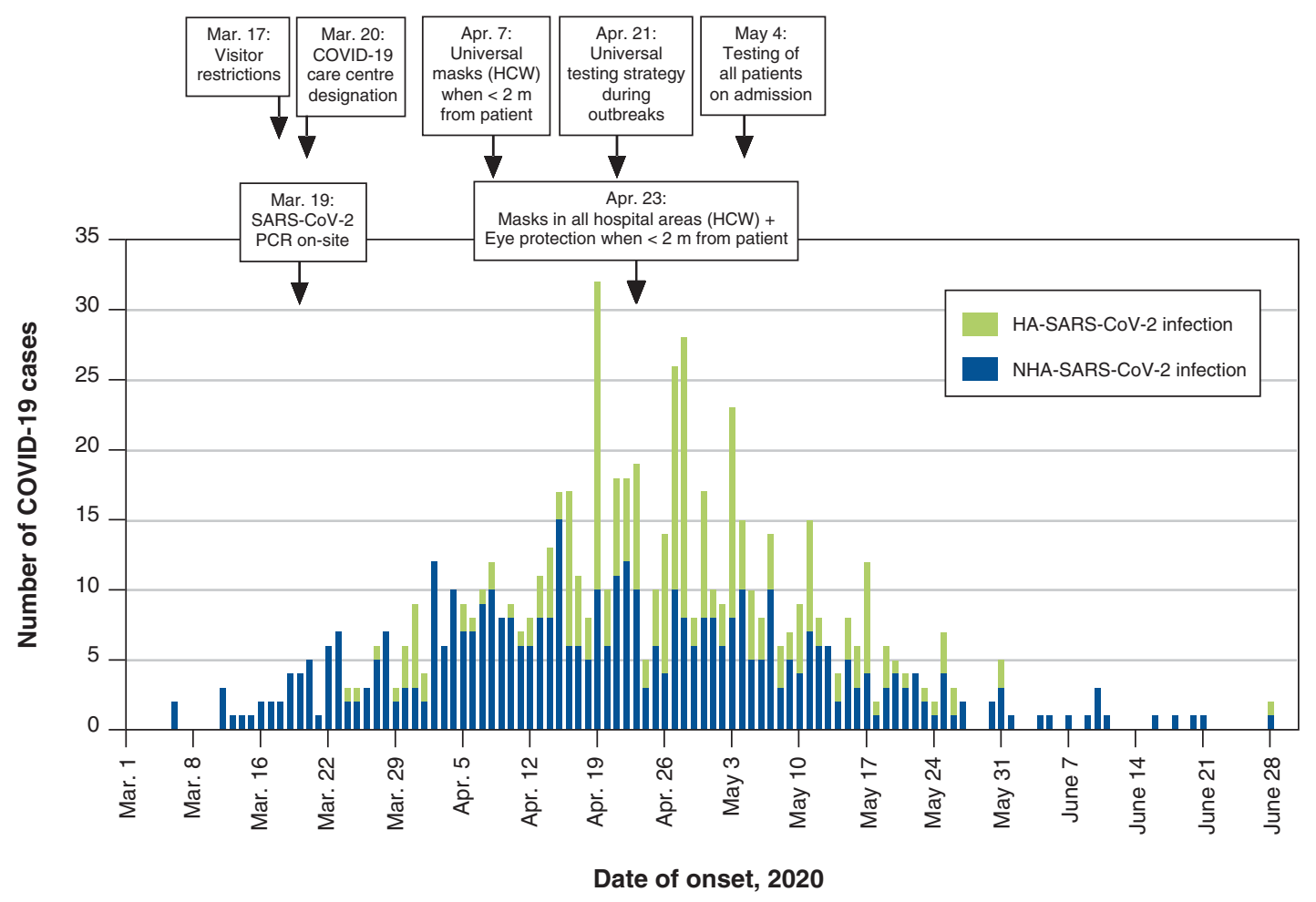

Figure 2: Epidemic curve of patients with SARS-CoV-2 infection in hospital. Note: HA = hospital-acquired, HCW = health care worker, NHA = non-hospital-acquired, PCR = polymerase chain reaction. 
Table 1: Baseline characteristics of patients with hospital-acquired and non-hospital-acquired SARS-CoV-2 infection

\begin{tabular}{|c|c|c|c|}
\hline Characteristic & $\begin{array}{l}\text { No. }(\%)^{*} \text { of hospital- } \\
\text { acquired cases } \\
n=253(36.3 \%)\end{array}$ & $\begin{array}{l}\text { No. }(\%)^{*} \text { of non-hospital- } \\
\text { acquired cases } \\
n=444(63.7 \%)\end{array}$ & $p$ value \\
\hline Age, yr, median (IQR) & $79(68-86)$ & $73(58.5-85)$ & \\
\hline Age, yr & & & 0.003 \\
\hline$<75$ & $98(38.7)$ & $231(52.0)$ & \\
\hline $75-84$ & $71(28.1)$ & $100(22.5)$ & \\
\hline$\geq 85$ & $84(33.2)$ & $113(25.5)$ & \\
\hline Female sex & $128(50.6)$ & $228(51.4)$ & 0.85 \\
\hline \multicolumn{4}{|l|}{ Comorbidity $†$} \\
\hline Moderate to severe chronic kidney disease $\ddagger$ & $35(13.8)$ & $52(11.7)$ & 0.42 \\
\hline Solid tumour & & & $<0.001$ \\
\hline Localized & $49(19.4)$ & $39(8.8)$ & \\
\hline Metastatic & $28(11.1)$ & $14(3.2)$ & \\
\hline Hematologic malignancy & $12(4.7)$ & $10(2.3)$ & 0.07 \\
\hline Diabetes & $87(34.4)$ & $156(35.1)$ & 0.84 \\
\hline COPD & $60(23.7)$ & $65(14.6)$ & 0.003 \\
\hline Myocardial infarction & $18(7.1)$ & $33(7.4)$ & 0.91 \\
\hline Dementia & $58(22.9)$ & $101(22.8)$ & 0.96 \\
\hline Charlson Comorbidity Index, median (IQR) & $2(1-4)$ & $1(0-3)$ & \\
\hline Provenance before admission§ & & & $<0.001$ \\
\hline Home & $180(71.2)$ & $237(53.4)$ & \\
\hline Long-term care facility & $9(3.6)$ & $49(11.0)$ & \\
\hline Others & $62(24.4)$ & $160(36.1)$ & \\
\hline Reason for admission & & & $<0.001$ \\
\hline Infectious & $70(27.9)$ & $7(1.6)$ & \\
\hline Nervous system & $38(15.1)$ & $7(1.6)$ & \\
\hline Neoplasm & $37(14.7)$ & $4(0.9)$ & \\
\hline Gastrointestinal & $21(8.4)$ & $9(2.0)$ & \\
\hline Other & $85(33.9)$ & $419(93.9)$ & \\
\hline Deprivation index of 4 or $5 \rrbracket$ & $150(59.3)$ & $243(54.7)$ & 0.16 \\
\hline Timing of diagnosis, $\mathrm{d}$ & & & $<0.001$ \\
\hline $0-6$ & $62(24.5)$ & $444(100)$ & \\
\hline $7-14$ & $91(36.0)$ & $0(0)$ & \\
\hline$>14$ & $100(39.5)$ & $0(0)$ & \\
\hline Treatment with corticosteroids & $46(18.2)$ & $66(14.9)$ & 0.25 \\
\hline Surgical procedure & $37(14.6)$ & $13(2.9)$ & 0.001 \\
\hline ICU admission & $21(8.3)$ & $85(19.1)$ & $<0.001$ \\
\hline ICU admission within 7 days & $17(81.0)$ & $74(87.1)$ & 0.47 \\
\hline Mechanical ventilation & $9(3.6)$ & $58(13.0)$ & 0.001 \\
\hline \multicolumn{4}{|c|}{$\begin{array}{l}\text { Note: } \mathrm{COPD}=\text { chronic obstructive pulmonary disease, ICU }=\text { intensive care unit, IQR = interquartile range, RPA = Résidence privée pour ainés (private } \\
\text { residences for older adults). } \\
\text { *Unless stated otherwise. } \\
\text { †Selected comorbidities from the Charlson Comorbidity Index. }{ }^{12} \\
\text { łModerate to severe chronic renal disease, defined as creatinine }>265 \mu \mathrm{mol} / \mathrm{L} \text {, as defined in the Charlson Comorbidity Index. }{ }^{12} \\
\text { §Others: congregate living settings such as RPA, intermediate and family-type resources (IR-FTR), transfer from another hospital and rehabilitation. } \\
\text { IThe deprivation index is based on patient postal code and } 2016 \text { census data. Results of various indicators are aggregated to create a deprivation index to } \\
\text { identify a deprived population. A score from } 1 \text { (least deprived) to } 5 \text { (most deprived) is calculated, with } 4 \text { and } 5 \text { signifying being deprived and most deprived. }{ }^{11}\end{array}$} \\
\hline
\end{tabular}




\begin{tabular}{|c|c|c|c|}
\hline Outcomes & $\begin{array}{l}\text { No. }(\%)^{*} \text { of hospital- } \\
\text { acquired cases } \\
n=253(36.3 \%)\end{array}$ & $\begin{array}{l}\text { No. }(\%)^{*} \text { of non- } \\
\text { hospital-acquired } \\
\text { cases } \\
n=444(63.7 \%)\end{array}$ & $p$ value \\
\hline Mortality by age, yr & $99(39.1)$ & $115(25.9)$ & 0.001 \\
\hline$<75$ & $29(29.6)$ & $23(10.0)$ & \\
\hline $75-84$ & $33(46.5)$ & $37(37.0)$ & \\
\hline$\geq 85$ & $37(44.1)$ & $55(48.7)$ & \\
\hline Hospital length of stay, median (IQR) & $25.0(15-46)$ & $8(3.5-16)$ & \\
\hline Disposition at discharge $\dagger$ & & & 0.001 \\
\hline Home & $74(48.1)$ & $197(59.9)$ & \\
\hline Long-term care facility & $32(20.8)$ & $73(22.2)$ & \\
\hline Rehabilitation & $26(16.9)$ & $20(6.1)$ & \\
\hline Others $\ddagger$ & $22(14.3)$ & $39(11.9)$ & \\
\hline Readmission within 90 days after discharge & $24(15.3)$ & $13(4.0)$ & 0.001 \\
\hline \multicolumn{4}{|c|}{$\begin{array}{l}\text { Note: IQR = interquartile range, RPA = Résidence privée pour ainés (private residences for older adults). } \\
\text { *Unless stated otherwise. } \\
\text { tDisposition at discharge was calculated only for survivors ( } n=154 \text { in hospital-acquired group and } n=329 \text { in non-hospital-acquired group). } \\
\text { fOthers: congregate living settings such as RPA, intermediate and family-type resources (IR-FTR) and transfer to another hospital or dedicated } \\
\text { centres for quarantine of patients with COVID-19. }\end{array}$} \\
\hline
\end{tabular}

chronic renal disease, solid metastatic cancer and hematologic malignancy were all associated with an increased odds of death. Conversely, being female seemed protective (OR 0.60, 95\% CI $0.42-0.87$ ). In the sensitivity analysis after excluding suspected HA cases, HA-SARS-CoV-2 infection status remained a significant risk factor for mortality (Appendix 1, eTable 2). No significant collinearity was detected between our covariates.

\section{Interpretation}

In the present study, one-third of all cases of SARS-CoV-2 infection in patients in hospital were acquired owing to inhospital transmission, with a higher mortality rate among patients younger than 75 years. Similar data from England reported that about $25 \%$ of COVID-19 cases in hospital were acquired during the hospital stay. ${ }^{16}$

A major factor influencing the prevalence of HA-SARS$\mathrm{CoV}-2$ infection is the hospital's infrastructure. Most of our wards have no mechanical ventilation systems and 2- and 4-bed rooms are separated by curtains. This design explains why one-third of patients sharing a room with a patient with COVID-19 subsequently tested positive for SARS-CoV-2 infection, even before the emergence of more contagious variants of concern. This proportion is consistent with household transmission rates between spouses $(37.8 \%$, 95\% CI $25.8 \%-$ $50.5 \%)^{17}$ and other reports on transmission between hospital roommates (up to $39 \%$ ). ${ }^{18,19}$

Although only 45/254 (17.7\%) of cases of HA-SARS$\mathrm{CoV}-2$ infection in our study were directly linked to transmission between patients in a multi-bed room, more HA cases

\begin{tabular}{|c|c|}
\hline Factors & OR $(95 \% \mathrm{Cl})$ \\
\hline \multicolumn{2}{|l|}{ HA cases, age, yr* } \\
\hline$<75$ & $2.78(1.44-5.38)$ \\
\hline $75-84$ & $1.25(0.64-2.42)$ \\
\hline$\geq 85$ & $0.75(0.41-1.35)$ \\
\hline \multicolumn{2}{|l|}{ Sex } \\
\hline Female & $0.60(0.42-0.87)$ \\
\hline \multicolumn{2}{|l|}{ Solid tumour† } \\
\hline Localized & $1.07(0.63-1.80)$ \\
\hline Metastatic & $5.91(2.84-12.30)$ \\
\hline Hematologic malignancy & $6.66(2.41-18.4)$ \\
\hline Moderate to severe chronic renal diseaseł & $2.96(1.74-5.04)$ \\
\hline Diabetes & $1.20(0.81-1.76)$ \\
\hline Chronic obstructive pulmonary disease & $1.02(0.64-1.62)$ \\
\hline Myocardial infarction & $0.90(0.47-1.74)$ \\
\hline $\begin{array}{l}\text { Note: } \mathrm{Cl}=\text { confidence interval, } \mathrm{HA}=\text { hospital-acquire } \\
\text { reference. } \\
\text { "Ref. }=\text { non-HA cases. } \\
\text { †Ref. }=\mathrm{y} \text {. } \\
\text { fDefined as creatinine }>265 \mu \mathrm{mol} / \mathrm{L} .\end{array}$ & R = odds ratio, Ref. $=$ \\
\hline
\end{tabular}

might be indirectly linked to high population density. ${ }^{7}$ The importance of crowding on COVID-19 incidence and mortality has already been shown in Canadian long-term care settings. ${ }^{20}$ Although difficult to quantify, various shortages (e.g., 
medical masks, PPE, hand sanitizer) might also have affected transmission rates. ${ }^{21,22}$ Nonetheless, as shown by Brigham and Women's Hospital's first-wave experience in Boston, Massachusetts, under optimal conditions, in-hospital transmission can be limited: only 1 case of COVID-19 was deemed to be hospital acquired among 697 hospital admissions. ${ }^{23}$ With new variants being of concern for increased transmissibility and potentially increased risk of death, it is even more important to reinforce nonpharmaceutical interventions to prevent nosocomial SARS-CoV-2 transmission. ${ }^{24,25}$

The mortality rate of patients with HA-SARS-CoV-2 infection in our study is also similar to that in existing data in the literature. ${ }^{4,7}$ Our NHA-SARS-CoV-2 infection group provided a good representation of patients with most severe HA-COVID-19, with $91.2 \%$ of the total NHA cases being community acquired and a mortality rate of $26.4 \%$. Nonetheless, patients with HA-SARS-CoV-2 infection still had a higher mortality rate. In a cohort study of 252 patients in hospital with active cancer and COVID-19, HA-COVID-19 was an independent risk factor of mortality (hazard ratio [HR] 2.3, $95 \%$ CI $1.3-4 ; p=0.005) .{ }^{26}$ The mortality rate of that study's HA-COVID-19 group was higher than in our results $(47 \% \mathrm{v}$. $38 \%$ ), likely because their cohort comprised exclusively patients with cancer.

Similarly, a history of metastatic cancer and hematologic malignancies were independent risk factors for death in our study. Therefore, the seriousness of the admission pathology has an important impact on patient outcome. A patient in hospital already weakened by an acute illness will have a difficult time managing the added physiologic stress of HASARS-CoV-2 infection. In another cohort study, HACOVID-19 was not a risk factor for death compared with NHA-COVID-19 (HR 0.71, 95\% CI 0.51-0.98). ${ }^{8}$ However, these authors did not stratify for age. The estimated risk of death for COVID-19 in adults aged 80 years and older in hospital is $60 \%$ irrespective of modes of acquisition. ${ }^{27}$ In our study, the increased risk of death from the inhospital acquisition of SARS-CoV-2 infection seemed to decrease with increasing age. This can be explained by an increase in comorbidities and frailty overwhelming the potential increased risk from HA-SARS-CoV-2 infections for older patients.

\section{Limitations}

There are limitations to our study. First, it is a single-centre retrospective study, which limits the generalizability of our transmission data and is also not meant to prove causality. Second, SARS-CoV-2 PCR testing capacity and indications changed quickly throughout the pandemic, potentially underestimating the prevalence of asymptomatic and paucisymptomatic cases of HA-SARS-CoV-2 infection. Third, there was no systematic postdischarge follow-up to assess whether patients developed COVID-19 after potential inhospital exposure, possibly underestimating HA-COVID-19 cases and SARS-CoV-2 infection by close contacts. Fourth, while our objective was to show the added mortality of concomitant illness on patients with SARS-CoV-2 infection, other potential confoundings associated with our classification might not be adequately adjusted by our regression model, possibly resulting in overestimation of the effect size. Also, lack of inter-rater reliability on data collection should be taken into consideration. Finally, combining proven and suspected cases might bias our results toward the null, therefore strengthening the positive results found in patients younger than 75 years.

\section{Conclusion}

For patients younger than 75 years, acquisition of SARSCoV-2 infection in hospital resulted in a higher risk of death than acquisition outside of hospital. Patients in hospital are vulnerable and at increased risk of complications if exposed to SARS-CoV-2. In-hospital viral transmission remains a major concern for patients, as many hospital design features are challenging to correct and circulating variants of SARSCoV-2 now seem more contagious. Even in the face of pandemic fatigue, these findings justify taking extraordinary measures to prevent COVID-19 hospital transmission and avoid preventable death.

\section{References}

1. Coronavirus disease (COVID-19) weekly epidemiological update and weekly operational update. Geneva: World Health Organization. Available: https:// www.who.int/emergencies/diseases/novel-coronavirus-2019/situation-reports / (accessed 2021 Sept. 6).

2. Population et composantes de l'accroissement démographique, Québec, 19712021. Québec: Institut de la statistique du Québec; updated 2021 Sept. 29. Available: https://statistique.quebec.ca/fr/document/population-et-structure-par-age-et -sexe-le-quebec/tableau/population-composantes-accroissement-demographique -quebec (accessed 2021 July 9).

3. COVID-19 daily epidemiology update. Ottawa: Government of Canada. Available: https://health-infobase.canada.ca/covid-19/epidemiological-summary -covid-19-cases.html (accessed 2021 Feb. 8).

4. Zhou F, Yu T, Du R, et al. Clinical course and risk factors for mortality of adult inpatients with COVID-19 in Wuhan, China: a retrospective cohort study. Lancet 2020;395:1054-62.

5. Wang D, Hu B, Hu C, et al. Clinical characteristics of 138 hospitalized patients with 2019 novel coronavirus-infected pneumonia in Wuhan, China. ZAMA 2020;323:1061-9.

6. Godoy P, Torner N, Soldevila N, et al. Hospital-acquired influenza infections detected by a surveillance system over six seasons, from 2010/2011 to 2015/2016. BMC Infect Dis 2020;20:80.

7. Rickman HM, Rampling T, Shaw K, et al. Nosocomial transmission of COVID-19: a retrospective study of 66 hospital-acquired cases in a London teaching hospital. Clin Infect Dis 2021;72:690-3.

8. Carter B, Collins JT, Barlow-Pay F, et al.; COPE Study Collaborators. Nosocomial COVID-19 infection: examining the risk of mortality. The COPE-Nosocomial Study (COVID in Older PEople). 7 Hosp Infect 2020; 106:376-84.

9. von Elm E, Altman DG, Egger M, et al.; STROBE Initiative. The Strengthening the Reporting of Observational studies in Epidemiology (STROBE) statement: guidelines for reporting observational studies. Int 7 Surg 2014;12:1495-9.

10. Harris PA, Taylor R, Thielke R, et al. Research electronic data capture (REDCap): a metadata-driven methodology and workflow process for providing translational research informatics support. F Biomed Inform 2009;42:377-81.

11. Guides méthodologiques. Québec: Institut national de santé publique du Québec. Available: https://www.inspq.qc.ca/defavorisation/indice-de -defavorisation-materielle-et-sociale/guides-methodologiques (accessed 2021 Feb. 8).

12. Deyo RA, Cherkin DC, Ciol MA. Adapting a clinical comorbidity index for use with ICD-9-CM administrative databases. 7 Clin Epidemiol 1992;45: 613-9.

13. SRAS-CoV-2: mesures de prévention et contrôle des infections pour les milieux de soins de courte durée — recommandations intérimaires. Québec: Institut national de santé publique du Québec; 2021. Available: https://www. inspq.qc.ca/publications/2906-pci-soins-aigus-covid19 (accessed 2021 Feb. 4). 
14. Khan KS, Reed-Embleton H, Lewis J, et al. Does nosocomial COVID-19 result in increased 30-day mortality? A multi-centre observational study to identify risk factors for worse outcomes in patients with COVID-19. 7 Hosp Infect 2021;107:91-4.

15. Nishiura H, Linton NM, Akhmetzhanov AR. Serial interval of novel coronavirus (COVID-19) infections. Int 7 Infect Dis 2020;93:284-6.

16. Discombe M. Covid infections caught in hospital rise by a third in one week. London (UK): Health Service Journal; 2020 Dec. 18. Available: https://www. hsj.co.uk/patient-safety/covid-infections-caught-in-hospital-rise-by-a-third-in -one-week/7029211.article (accessed 2021 Jan. 27).

17. Madewell ZJ, Yang Y, Longini IM Jr, et al. Household transmission of SARSCoV-2: a systematic review and meta-analysis. 7AMA Netw Open 2020;3:e2031756.

18. Karan A, Klompas M, Tucker R, et al.; CDC Prevention Epicenters Program. The risk of SARS-CoV-2 transmission from patients with undiagnosed COVID-19 to roommates in a large academic medical center. Clin Infect Dis 2021 June 17 [Epub ahead of print]. doi: 10.1093/cid/ciab564.

19. Chow K, Aslam A, McClure T, et al. Risk of healthcare-associated transmission of SARS-CoV-2 in hospitalized cancer patients. Clin Infect Dis 2021 July 30 [Epub ahead of print]. doi: 10.1093/cid/ciab670.

20. Brown KA, Jones A, Daneman N, et al. Association between nursing home crowding and COVID-19 infection and mortality in Ontario, Canada. FAMA Intern Med 2021;181:229-36.

21. Richterman A, Meyerowitz EA, Cevik M. Hospital-acquired SARS-CoV-2 infection: lessons for public health. FAMA 2020;324:2155-6.

22. Oliver D. David Oliver: Could we do better on hospital acquired COVID-19 in a future wave? BMF 2021;372.

23. Rhee C, Baker M, Vaidya V, et al.; CDC Prevention Epicenters Program. Incidence of nosocomial COVID-19 in patients hospitalized at a large US academic medical center. 7AMA Netw Open 2020;3:e2020498.

24. Galloway SE, Paul P, MacCannell DR, et al. Emergence of SARS-CoV-2 B.1.1.7 lineage: United States, December 29, 2020-January 12, 2021. MMWR Morb Mortal Wkly Rep 2021;70:95-9.

25. Horby P, Huntley C, Davies N, et al. NERVTAG paper on COVID-19 variant of concern B.1.1.7. London (UK): Public Health England; 2021. Available: https://www.gov.uk/government/publications/nervtag-paper-on-covid-19-variant -of-concern-b117 (accessed 2021 Feb. 8).

26. Elkrief A, Desilets A, Papneja N, et al. High mortality among hospitalacquired COVID-19 infection in patients with cancer: a multicentre observational cohort study. Eur 7 Cancer 2020;139:181-7.

27. Brill SE, Jarvis HC, Ozcan E, et al. COVID-19: a retrospective cohort study with focus on the over-80s and hospital-onset disease. BMC Med 2020;18:194.
Affiliations: Pulmonary Division (Melançon, Brosseau), Department of Medicine, Hôpital Maisonneuve-Rosemont, Centre intégré universitaire de santé et de services sociaux (CIUSSS) de l'Est-de-l'Île-deMontréal; Department of Medicine (Melançon, Brosseau, Bartoli, Wang), Faculty of Medicine, Université de Montréal; Critical Care Division (Brosseau, Wang), Internal Medicine Division (Bartoli), and Microbiology and Infectious Disease Division (Labbé, Lavallée, Marchand-Senécal), Department of Medicine, Hôpital MaisonneuveRosemont, CIUSSS de l'Est-de-l'̂̂le-de-Montréal; Department of Microbiology, Infectious Diseases and Immunology (Labbé, Lavallée, Marchand-Senécal), Faculty of Medicine, Université de Montréal, Montréal, Que.

Contributors: All of the authors contributed to the conception and design of the work, and the acquisition, analysis, and interpretation of data. All of the authors drafted the manuscript, revised it critically for important intellectual content, gave final approval of the version to be published and agreed to be accountable for all aspects of the work.

Content licence: This is an Open Access article distributed in accordance with the terms of the Creative Commons Attribution (CC BYNC-ND 4.0) licence, which permits use, distribution and reproduction in any medium, provided that the original publication is properly cited, the use is noncommercial (i.e., research or educational use), and no modifications or adaptations are made. See: https://creativecommons. org/licenses/by-nc-nd/4.0/

Data sharing: The complete data set is available to other researchers after request to the corresponding author and upon adequate justification. Secure transfer of the data set can be established after approval.

Acknowledgements: The authors thank the hospital Infection Prevention and Control (IPAC) team for its valiant efforts in controlling the viral propagation in the hospital, particularly Chantal Bellerose, Chief of IPAC division, for her support in gathering data and timeline of events. The authors also thank Stéphanie Beauchemin for coordinating the Research Ethics Board submission and data collection.

Supplemental information: For reviewer comments and the original submission of this manuscript, please see www.cmajopen.ca/content /10/1/E74/suppl/DC1. 